Theoretical Mathematics \& Applications, Vol. 11, No. 3, 2021, 1-16

ISSN: 1792-9687(print), 1792-9709(online)

https://doi.org/10.47260/tma/1131

Scientific Press International Limited

\title{
A Note on Variational Principle of Subsets for Nonautonomous Dynamical Systems
}

\author{
Jiao Yang ${ }^{1}$
}

\begin{abstract}
In this paper, we introduce measure-theoretic for Borel probability measures to characterize upper and lower Katok measure-theoretic entropies in discrete type and the measure-theoretic entropy for arbitrary Borel probability measure in nonautonomous case. Then we establish new variational principles for Bowen topological entropy for nonautonomous dynamical systems.
\end{abstract}

JEL classification numbers: 37A35.

Keywords: Nonautonomous, Measure-theoretical entropies, Variational principles.

${ }^{1}$ School of Mathematical Sciences, Nanjing Normal University, Nanjing 210023, Jiangsu, P.R.China.

Article Info: Received: September 26, 2021. Revised: October 22, 2021.

Published online: October 31, 2021. 


\section{Introduction}

As an important invariant of topological conjugacy, the notion of topological entropy was introduced by Adler, Konheim and McAndrew [1] in 1965 [3]. Topological entropy is a key tool to measure the complexity of a classical dynamical system, i.e. the exponential growth rate of the number of distinguishable orbits of the iterates of an endomorphism of a compact metric space. In 1973, Bowen [2] introduced the topological entropy $h_{\text {top }}^{B}(T, Z)$ for any set $Z$ in a topological dynamical system $X$, in a way resembling Hausdorff dimension, where $\mathrm{X}$ is a compact metric space and $T: X \rightarrow X$ is a continuous self-map. Bowen topological entropy plays a key role in topological dynamics and dimension theory [2]. In 2012, Feng and Huang [6] showed that there is certain variational relation between Bowen topological entropy and measure-theoretic entropy for arbitrary non-invariant compact set of a topological dynamical system $(X, T)$.

Following the idea of Brin and Katok [8], they defined the measure-theoretic entropy for Borel probability measure on $\mathrm{X}$ for their results.

In contrast with the autonomous discrete, in contrast with the autonomous discrete case [12], the properties of the entropies for the nonautonomous dynamical systems have not been fully investigated. In order to have a good understanding of the topological entropy of a skew product of dynamical systems (as we know that the calculation of its topological entropy can be transformed into that of its fibers), Kolyada and Snoha [4] proposed the concept of topological entropy in 1996 for a nonautonomous dynamical system determined by a sequence of maps.

A nonautonomous discrete dynamical system (in short: NADDS) is a natural generalization of classical dynamical systems, its dynamics are determined by a sequence of continuous self-maps $f_{n}: X \rightarrow X$ where $n \in \mathbf{N}$, defined on a compact metric space $X$.

By a nonautonomous dynamical system (NADDS for short) we understand a pair $\left(X,\left\{f_{n}\right\}_{n=1}^{\infty}\right)$, where $X$ is a compact metric space endowed with a metric $d$ and $\left\{f_{n}\right\}_{n=1}^{\infty}$, is a sequence of continuous maps from $X$ to $X$. In 2013, Kawan [10] generalized the classical notion of measure-theoretical entropy established by Kolomogorov and Sinai to NADSs, and proved that the measure-theoretical entropy can be estimated from above by its topological entropy. Following the idea of Brin and Katok [8] and Zhou [7] introduced the measure-theoretical entropy in nonautonomous case and established a variational principle for the first time. More results related to entropy for NADSs were developed in [11]. In this paper, We introduce ideas of Wang [9] to nonautonomous systems to establish new variational principles for Bowen topological entropy for nonautonomous dynamical systems. 
Give a NADDS $\left(X,\left\{f_{n}\right\}_{n=1}^{\infty}\right)$. For each $n \in \mathbf{N}^{+}$, the Bowen metric $d_{n}$ on $X$ is defined by $d_{n}(x, y)=\max _{0 \leq i \leq n-1} d\left(f_{1}^{i}(x), f_{1}^{i}(y)\right)$. For every $\varepsilon>0$, we denote by $B_{n}(x, \varepsilon)$ the open ball of radius $\varepsilon$ in the metric $d_{n}$ around $x$, i.e., $B_{n}(x, \varepsilon)=\left\{y \in X: d_{n}(x, y)<\varepsilon\right\}$.

We also consider a nonautonomous dynamical system (for short NADS) $(X, \phi)$ where $(X, d)$ is a compact metric space and $\phi:[0, \infty) \times X \rightarrow X$ is a continuous map with $\phi(0, x)=x$ for $x \in X$. We want to know whether there is certain variational relation of entropy for nonautonomous dynamical systems. For our study, we need to define the measure-theoretic entropy for arbitrary Borel probability measure in nonautonomous case.

Given a NADS $(X, \phi)$. For any $t \in[0, \infty)$, the $t$ Bowen metric $d_{t}^{\phi}$ on $X$ is defined by

$$
d_{t}^{\phi}(x, y)=\max _{0 \leq i \leq n-1} d(\phi(s, x), \phi(s, y))
$$

For every $\varepsilon>0$, we denote by $B_{t}^{\phi}(x, \varepsilon)$ the open ball of radius $\varepsilon$ in the metric $d_{t}^{\phi}$ around $x$, i.e.,

$$
B_{t}^{\phi}(x, \varepsilon)=\left\{y \in X: d_{t}^{\phi}(x, y)<\varepsilon\right\} .
$$

Write $\phi^{i}(x):=\phi(i, x)$ for $i=1,2, \cdots$ and $x \in X$.

In this case, we take $f_{n}(x)=\phi^{n}(x)$, then $\left\{\phi^{n}\right\}_{n=1}^{\infty}$ is a NADDS.

Let $M(X)$ denote the set of all Borel probability measures on $X, Z \subset X$ and $\mu \in M(X),\left(X,\left\{f_{n}\right\}_{n=1}^{\infty}\right)$ is a NADDS.

(1) A set $E \subset Z$ is said to be an $(n, \varepsilon, Z)$-separated set if $x, y \in E$ with $x \neq y$ implies $d_{n}^{\phi}(x, y)>\varepsilon$. Let $r_{n}(\varepsilon, Z)$ denote the maximum cardinality of $(n, \varepsilon, Z)$ -separated set.

(2) A set $F \subset Z$ is said to be an $(n, \varepsilon, Z)$-spanning set if, for any $x \in X$, there exists $y \in F$ with $d_{n}^{\phi}(x, y) \leq \varepsilon$. Let $s_{n}(\varepsilon, Z)$ denote the minimum cardinality of $(n, \varepsilon, Z)$-spanning sets.

(3) A set $F \subset Z$ is said to be a $(\mu, n, \varepsilon, \delta)$-spanning set if the union $\bigcup_{x \in F} B_{n}(x, \varepsilon)$ has $\mu$-measure more than or equal to $1-\delta$. Let $r_{n}(\mu, \varepsilon, \delta)$ denote the minimum cardinality of $(\mu, n, \varepsilon, \delta)$-spanning sets. 
(4) We introduce a useful set: $X_{\mu, \delta}=\{Z \subset X: \mu(Z) \geq 1-\delta\}$.

Then it is clear that

$$
r_{n}(\mu, \varepsilon, \delta)=\inf _{Z \in X_{\mu, \delta}} r_{n}(\varepsilon, Z)
$$

An open cover of $X$ is a family of open subsets of $X$, whose union is $X$. For two covers $\mathrm{U}$ and $\mathrm{V}$ we say that $\mathrm{U}$ is a refinement of $\mathrm{V}$ if for each $U \in \mathrm{U}$ there is $V \in \mathrm{V}$ with $U \in V$. For $n \in \mathbf{N}$ and open covers $\mathrm{U}_{1} /, \mathrm{U}_{2}, \cdots, \mathrm{U}_{n} /$ of $X$ we denote

$$
\bigvee_{i=1}^{n} \mathrm{U}_{i}=\left\{A_{1} \cap A_{2} \cap \cdots \cap A_{n}: A_{1} \in \mathrm{U}_{1}, A_{2} \in \mathrm{U}_{2}, \cdots, A_{n} \in \mathrm{U}_{n}\right\}
$$

Note that $\vee_{i=1}^{n} \mathrm{U}_{i}$ is also an open cover of $X$. We denote by $\mathrm{N}(\mathrm{U})$ the minimal cardinality of all subcovers chosen from $U$.

Set

$$
f_{i}^{0}=i d_{X}, f_{i}^{n}=f_{i+(n-1)} \circ f_{i+(n-2)} \circ \cdots \circ f_{i+1} \circ f_{i}, f_{i}^{-n}=\left(f_{i}^{n}\right)^{-1}
$$

For all $i, n \in \mathbf{N}$, where $i d_{X}$ is the identity map on $X$.

Let

$$
h_{\text {top }}\left(\left\{f_{n}\right\}_{n=1}^{\infty}, \mathrm{U}\right)=\limsup _{n \rightarrow \infty} \frac{\log \mathrm{N}\left(\bigvee_{i=0}^{n-1} f_{1}^{-i} \mathrm{U}_{i}\right)}{n} .
$$

The topological entropy is defined by

$$
h_{\text {top }}\left(X,\left\{f_{n}\right\}_{n=1}^{\infty}\right)=\left\{h_{\text {top }}\left(\left\{f_{n}\right\}_{n=1}^{\infty}, \mathrm{U}\right): \mathrm{U} \text { is an open cover of } X\right\} \text {. }
$$

It was proved in $\backslash$ cite $\{\mathrm{AKM}\}$ that for every NADS, we have $h_{\text {top }}\left(X,\left\{f_{n}\right\}_{n=1}^{\infty}\right)=\lim _{\varepsilon \rightarrow 0} \limsup _{n \rightarrow \infty} \frac{\log s_{n}(\varepsilon, X)}{n}=\lim _{\varepsilon \rightarrow 0} \limsup _{n \rightarrow \infty} \frac{\log r_{n}(\varepsilon, X)}{n}$.

Following the idea of Katok $\backslash$ cite $\{$ AKM $\}$, we give the following.

Let $\mu \in M(X)$. The NADDS Katok measure-theoretical lower and upper entropies of $\mu$ are defined respectively by

$$
\begin{aligned}
& \underline{h}^{K}\left(\left\{f_{n}\right\}_{n=1}^{\infty}\right)=\lim _{\delta \rightarrow 0} \lim _{\varepsilon \rightarrow 0} \liminf _{n \rightarrow \infty} \frac{1}{n} \log r_{n}(\mu, \varepsilon, \delta) \\
& \bar{h}_{\mu}^{K}\left(\left\{f_{n}\right\}_{n=1}^{\infty}\right)=\lim _{\delta \rightarrow 0} \lim _{\varepsilon \rightarrow 0} \limsup _{n \rightarrow \infty} \frac{1}{n} \log r_{n}(\mu, \varepsilon, \delta)
\end{aligned}
$$


In this paper, we introduce many quantities for Borel probability measure $\mu \in M(X)$, respectively denoted by $e_{\mu}\left(\left\{f_{n}\right\}_{n=1}^{\infty}\right), \quad \underline{e}_{\mu}\left(\left\{f_{n}\right\}_{n=1}^{\infty}\right), \bar{e}_{\mu}\left(\left\{f_{n}\right\}_{n=1}^{\infty}\right)$, $e_{\mu}^{*}\left(\left\{f_{n}\right\}_{n=1}^{\infty}\right)$, and so on.

According to the relations of the several types of NADS topological entropies, it is natural to consider relationship of some new quantities and Katok measuretheoretical lower and upper entropies. Therefore, we have the first main result.

\section{Main Results}

Theorem 2.1 Let $\left(X,\left\{f_{n}\right\}_{n=1}^{\infty}\right)$ be a NADDS, $\mu \in M(X)$.

Then following statements hold.

(1) For any $Z \subseteq X, h_{\text {top }}^{B}\left(\left\{f_{n}\right\}_{n=1}^{\infty}, Z\right) \leq h_{\text {top }}^{P}\left(\left\{f_{n}\right\}_{n=1}^{\infty}, Z\right)$.

(2) $\bar{h}_{\mu}^{K}\left(\left\{f_{n}\right\}_{n=1}^{\infty}\right)=\bar{e}_{\mu}\left(\left\{f_{n}\right\}_{n=1}^{\infty}\right)$.

(3) $\underline{h}_{\mu}^{K}\left(\left\{f_{n}\right\}_{n=1}^{\infty}\right)=\underline{-e}_{\mu}\left(\left\{f_{n}\right\}_{n=1}^{\infty}\right)$.

(4) $e_{\mu}\left(\left\{f_{n}\right\}_{n=1}^{\infty}\right) \leq \underline{e}_{\mu}\left(\left\{f_{n}\right\}_{n=1}^{\infty}\right) \leq \bar{e}_{\mu}\left(\left\{f_{n}\right\}_{n=1}^{\infty}\right)$.

(5) $e_{\mu}\left(\left\{f_{n}\right\}_{n=1}^{\infty}\right) \leq e_{\mu}^{*}\left(\left\{f_{n}\right\}_{n=1}^{\infty}\right)=\lim _{\varepsilon \rightarrow 0} \lim _{\delta \rightarrow 0} \inf _{Z \in X_{\mu, \delta}} h_{\text {top }}^{P}\left(\left\{f_{n}\right\}_{n=1}^{\infty}, Z, \varepsilon\right)$.

where the definitions of these notions will be given in Section 3.

Theorem 2.2 Let $\left(X,\left\{f_{n}\right\}_{n=1}^{\infty}\right)$ be a $N A D D S$. If $K \subset X$ is a non-empty and compact, then

$$
h_{\text {top }}^{B}\left(\left\{f_{n}\right\}_{n=1}^{\infty}, K\right)=\sup \left\{e_{\mu}\left(\left\{f_{n}\right\}_{n=1}^{\infty}\right): \mu \in M(X), \mu(K)=1\right\} .
$$

Theorem 2.3 Let $(X, \phi)$ be a NADS, $\mu \in M(X)$. Then following statements hold.

(1) For any $Z \subseteq X, h_{\text {top }}^{B}(\phi, Z) \leq h_{\text {top }}^{P}(\phi, Z)$.

(2) $e_{\mu}(\phi) \leq \underline{-e}_{\mu}(\phi) \leq \bar{e}_{\mu}(\phi)$.

(3) $e_{\mu}(\phi) \leq e_{\mu}^{*}(\phi)=\lim _{\varepsilon \rightarrow 0} \lim _{\delta \rightarrow 0} \inf _{Z \in X_{\mu, \delta}} h_{\text {top }}^{P}(\phi, Z, \varepsilon)$. 
Theorem 2.4 Let $(X, \phi)$ be a NADS. If $K \subset X$ is non-empty and compact, then $h_{\text {top }}^{B}(\phi, K)=\sup \left\{e_{\mu}(\phi): \mu \in M(X), \mu(K)=1\right\}$.

\section{Preliminary Notes}

\subsection{NADDS}

In this subsection, let $\left(X,\left\{f_{n}\right\}_{n=1}^{\infty}\right)$ be a NADDS, next we introduced NADDS's entropies. Following, we give some definitions of several NADDS topological entropies of subsets.

Definition 3.1 Let $Z \subset X, s \geq 0, N \in \mathbf{N}$ and $\varepsilon>0$, define

$$
M_{N, \varepsilon}^{s}\left(\left\{f_{n}\right\}_{n=1}^{\infty}, Z\right)=\inf \sum_{i} \exp \left(-s n_{i}\right),
$$

where the infimum is taken over all finite or countable families $\left\{B_{n_{i}}\left(x_{i}, \varepsilon\right)\right\}$ such that $x_{i} \in X, \quad n_{i} \geq N$ and $\bigcup_{i} B_{n_{i}}\left(x_{i}, \varepsilon\right) \supseteq Z$. The quantity $M_{N, \varepsilon}^{s}\left(\left\{f_{n}\right\}_{n=1}^{\infty}, Z\right)$ does not decrease as $N$ increase and $\varepsilon$ decreases, hence the following limits exist:

$$
\begin{aligned}
& M_{\varepsilon}^{s}\left(\left\{f_{n}\right\}_{n=1}^{\infty}, Z\right)=\lim _{N \rightarrow \infty} M_{N, \varepsilon}^{s}\left(\left\{f_{n}\right\}_{n=1}^{\infty}, Z\right), \\
& \left.M^{s}\left(\left\{f_{n}\right\}_{n=1}^{\infty}, Z\right)=\lim _{\varepsilon \rightarrow 0} M_{\varepsilon}^{s}\left(\left\{f_{n}\right\}_{n=1}^{\infty}, Z\right)\right) .
\end{aligned}
$$

Bowen's topological entropy $h_{\text {top }}^{B}\left(\left\{f_{n}\right\}_{n=1}^{\infty}, Z\right)$ is defined as a critical value of the parameters $s$, where $M^{s}\left(\left\{f_{n}\right\}_{n=1}^{\infty}, Z\right)$ jumps from $\infty$ to 0 , i.e.

$$
M^{s}\left(\left\{f_{n}\right\}_{n=1}^{\infty}, Z\right)= \begin{cases}0, & , s>h_{\text {top }}^{B}\left(\left\{f_{n}\right\}_{n=1}^{\infty}, Z\right) \\ \infty, & , s<h_{\text {top }}^{B}\left(\left\{f_{n}\right\}_{n=1}^{\infty}, Z\right)\end{cases}
$$

Definition 3.2 Let $Z \subset X$. For $s \geq 0, N \in \mathbf{N}$ and $\varepsilon>0$, define

$$
P_{N, \varepsilon}^{s}\left(\left\{f_{n}\right\}_{n=1}^{\infty}, Z\right)=\sup \sum_{i} \exp \left(-s n_{i}\right),
$$

where the supremum is taken over all finite or countable pairwise disjoint families $\left\{\bar{B}_{n_{i}}\left(x_{i}, \varepsilon\right)\right\}$ such that $x_{i} \in Z, \quad n_{i} \geq N$ for all $i$,

where

$$
\bar{B}_{n_{i}}\left(x_{i}, \varepsilon\right):=\left\{y \in X: d_{n}(x, y) \leq \varepsilon\right\} \text {. }
$$


The quantity $P_{N, \varepsilon}^{s}\left(\left\{f_{n}\right\}_{n=1}^{\infty}, Z\right)$ does not decrease as $N, \varepsilon$ decrease.

Hence the following limit exists:

$$
P_{\varepsilon}^{s}\left(\left\{f_{n}\right\}_{n=1}^{\infty}, Z\right)=\lim _{N \rightarrow \infty} P_{N, \varepsilon}^{s}\left(\left\{f_{n}\right\}_{n=1}^{\infty}, Z\right)
$$

Define

$$
\mathrm{P}_{\varepsilon}^{s}\left(\left\{f_{n}\right\}_{n=1}^{\infty}, Z\right)=\inf \left\{\sum_{i=1}^{\infty} P_{\varepsilon}^{s}\left(\left\{f_{n}\right\}_{n=1}^{\infty}, Z_{i}\right): \bigcup_{i=1}^{\infty} Z_{i} \supseteq Z\right\} .
$$

There exists a critical value of the parameters $s$, which we will denote by $h_{\text {top }}^{P}\left(\left\{f_{n}\right\}_{n=1}^{\infty}, Z, \varepsilon\right)$, where $\mathrm{P}_{\varepsilon}^{s}\left(\left\{f_{n}\right\}_{n=1}^{\infty}, Z\right)$ jumps from $\infty$ to 0 , i.e.

$$
\mathrm{P}^{s}\left(\left\{f_{n}\right\}_{n=1}^{\infty}, Z\right)=\left\{\begin{array}{l}
0, s>h_{\text {top }}^{P}\left(\left\{f_{n}\right\}_{n=1}^{\infty}, Z, \varepsilon\right) \\
\infty, s<h_{\text {top }}^{P}\left(\left\{f_{n}\right\}_{n=1}^{\infty}, Z, \varepsilon\right)
\end{array}\right.
$$

Note that $h_{\text {top }}^{P}\left(\left\{f_{n}\right\}_{n=1}^{\infty}, Z, \varepsilon\right)$ increases when $\varepsilon$ decreases.

We call

$$
h_{\text {top }}^{P}\left(\left\{f_{n}\right\}_{n=1}^{\infty}, Z\right):=\lim _{\varepsilon \rightarrow 0} h_{\text {top }}^{P}\left(\left\{f_{n}\right\}_{n=1}^{\infty}, Z, \varepsilon\right)
$$

the topological packing entropy of $Z$.

Definition 3.3 Let $Z \subseteq X$. For $s \geq 0, N \in \mathbf{N}$ and $\varepsilon>0$, define

$$
R_{N, \varepsilon}^{s}\left(\left\{f_{n}\right\}_{n=1}^{\infty}, Z\right)=\inf \sum_{i} \exp (-s N)
$$

where the infimum is taken over all finite or countable families $\left\{B_{N}\left(x_{i}, \varepsilon\right)\right\}$ such that $x_{i} \in X$, and $\bigcup_{i} B_{N}\left(x_{i}, \varepsilon\right) \supseteq Z$.

Let

$$
\begin{aligned}
& \underline{R}_{\varepsilon}^{s}\left(\left\{f_{n}\right\}_{n=1}^{\infty}, Z\right)=\liminf _{N \rightarrow \infty} R_{N, \varepsilon}^{s}\left(\left\{f_{n}\right\}_{n=1}^{\infty}, Z\right), \\
& \bar{R}_{\varepsilon}^{s}\left(\left\{f_{n}\right\}_{n=1}^{\infty}, Z\right)=\underset{N \rightarrow \infty}{\limsup } R_{N, \varepsilon}^{s}\left(\left\{f_{n}\right\}_{n=1}^{\infty}, Z\right)
\end{aligned}
$$

and

$$
\begin{aligned}
& \underline{C h}_{Z}\left(\left\{f_{n}\right\}_{n=1}^{\infty}, \varepsilon\right)=\inf \left\{s: \underline{R}_{\varepsilon}^{s}\left(\left\{f_{n}\right\}_{n=1}^{\infty}, Z\right)=0\right\}=\sup \left\{s: \underline{R}_{\varepsilon}^{s}\left(\left\{f_{n}\right\}_{n=1}^{\infty}, Z\right)=+\infty\right\}, \\
& \overline{C h}_{Z}\left(\left\{f_{n}\right\}_{n=1}^{\infty}, \varepsilon\right)=\inf \left\{s: \bar{R}_{\varepsilon}^{s}\left(\left\{f_{n}\right\}_{n=1}^{\infty}, Z\right)=0\right\}=\sup \left\{s: \bar{R}_{\varepsilon}^{s}\left(\left\{f_{n}\right\}_{n=1}^{\infty}, Z\right)=+\infty\right\} .
\end{aligned}
$$


The lower and upper capacity topological entropies of $\left\{f_{n}\right\}_{n=1}^{\infty}$ restricted to $Z$ are defined respectively by

$$
\begin{aligned}
& \underline{C h}_{Z}\left(\left\{f_{n}\right\}_{n=1}^{\infty}\right)=\lim _{\varepsilon \rightarrow 0} \operatorname{Ch}\left(\left\{f_{n}\right\}_{n=1}^{\infty}, \varepsilon\right), \\
& \overline{C h} h_{Z}\left(\left\{f_{n}\right\}_{n=1}^{\infty}\right)=\lim _{\varepsilon \rightarrow 0} \bar{C} h_{Z}\left(\left\{f_{n}\right\}_{n=1}^{\infty}, \varepsilon\right) .
\end{aligned}
$$

Definition 3.4 Let $\mu \in M(X), \quad s \geq 0, \quad N \in \mathbf{N}, \quad \varepsilon>0$ and $0<\delta<1$, define

$$
M_{N, \varepsilon}^{s}\left(\left\{f_{n}\right\}_{n=1}^{\infty}, \mu, \delta\right)=\inf \sum_{i} \exp \left(-s n_{i}\right),
$$

where the infimum is taken over all finite or countable families $\left\{B_{n_{i}}\left(x_{i}, \varepsilon\right)\right\}$ such that $x_{i} \in X, n_{i} \geq N$ and $\mu\left(\bigcup_{i} B_{n_{i}}\left(x_{i}, \varepsilon\right)\right) \geq 1-\delta$.

The quantity $M_{N, \varepsilon}^{s}\left(\left\{f_{n}\right\}_{n=1}^{\infty}, \mu, \delta\right)$ does not decrease as $N$ increase, hence the following limit exist:

$$
M_{\varepsilon}^{s}\left(\left\{f_{n}\right\}_{n=1}^{\infty}, \mu, \delta\right)=\lim _{N \rightarrow \infty} M_{N, \varepsilon}^{s}\left(\left\{f_{n}\right\}_{n=1}^{\infty}, \mu, \delta\right)
$$

Using standard method, we have following is well- defined:

$e_{\mu}\left(\left\{f_{n}\right\}_{n=1}^{\infty}, \varepsilon, \delta\right)=\inf \left\{s: M_{\varepsilon}^{s}\left(\left\{f_{n}\right\}_{n=1}^{\infty}, \mu, \delta\right)=0\right\}=\sup \left\{s: M_{\varepsilon}^{s}\left(\left\{f_{n}\right\}_{n=1}^{\infty}, \mu, \delta\right)=+\infty\right\}$

Defined

$$
e_{\mu}\left(\left\{f_{n}\right\}_{n=1}^{\infty}\right)=\lim _{\delta \rightarrow 0} \lim _{\varepsilon \rightarrow 0} e_{\mu}\left(\left\{f_{n}\right\}_{n=1}^{\infty}, \varepsilon, \delta\right)
$$

Definition 3.5 Let $\mu \in M(X), s \geq 0, N \in \mathbf{N}, \quad \varepsilon>0$ and $0<\delta<1$, put

$$
R_{N, \varepsilon}^{s}\left(\left\{f_{n}\right\}_{n=1}^{\infty}, \mu, \delta\right)=\inf \sum_{i} \exp (-s N),
$$

where the infimum is taken over all finite or countable families $\left\{B_{N}\left(x_{i}, \varepsilon\right)\right\}$ such that $x_{i} \in X$, and $\mu\left(\bigcup_{i} B_{N}\left(x_{i}, \varepsilon\right)\right) \geq 1-\delta$.

Let

$$
\begin{aligned}
& \underline{R}_{\varepsilon}^{s}\left(\left\{f_{n}\right\}_{n=1}^{\infty}, \mu, \delta\right)=\liminf _{N \rightarrow \infty} R_{N, \varepsilon}^{s}\left(\left\{f_{n}\right\}_{n=1}^{\infty}, \mu, \delta\right), \\
& \bar{R}_{\varepsilon}^{s}\left(\left\{f_{n}\right\}_{n=1}^{\infty}, \mu, \delta\right)=\limsup _{N \rightarrow \infty} R_{N, \varepsilon}^{s}\left(\left\{f_{n}\right\}_{n=1}^{\infty}, \mu, \delta\right) .
\end{aligned}
$$


Using standard method, we have following is well- defined:

$$
\begin{aligned}
& \underline{e}_{\mu}\left(\left\{f_{n}\right\}_{n=1}^{\infty}, \varepsilon, \delta\right)=\inf \left\{s: \underline{R}_{\varepsilon}^{s}\left(\left\{f_{n}\right\}_{n=1}^{\infty}, \mu, \delta\right)=0\right\}=\sup \left\{s: \underline{R}_{\varepsilon}^{s}\left(\left\{f_{n}\right\}_{n=1}^{\infty}, \mu, \delta\right)=+\infty\right\} \\
& \bar{e}_{\mu}\left(\left\{f_{n}\right\}_{n=1}^{\infty}, \varepsilon, \delta\right)=\inf \left\{s: \bar{R}_{\varepsilon}^{s}\left(\left\{f_{n}\right\}_{n=1}^{\infty}, \mu, \delta\right)=0\right\}=\sup \left\{s: \bar{R}_{\varepsilon}^{s}\left(\left\{f_{n}\right\}_{n=1}^{\infty}, \mu, \delta\right)=+\infty\right\}
\end{aligned}
$$

Define

$$
\begin{aligned}
& \underline{e}_{\mu}\left(\left\{f_{n}\right\}_{n=1}^{\infty}\right)=\lim _{\delta \rightarrow 0} \lim _{\varepsilon \rightarrow 0} e_{\mu}\left(\left\{f_{n}\right\}_{n=1}^{\infty}, \varepsilon, \delta\right), \\
& \bar{e}_{\mu}\left(\left\{f_{n}\right\}_{n=1}^{\infty}\right)=\lim _{\delta \rightarrow 0} \lim _{\varepsilon \rightarrow 0} \bar{e}_{\mu}\left(\left\{f_{n}\right\}_{n=1}^{\infty}, \varepsilon, \delta\right) .
\end{aligned}
$$

Definition 3.6 Let $\mu \in M(X), \quad s \geq 0, \quad N \in \mathbf{N}, \quad \varepsilon>0$ and $0<\delta<1$, put

$$
\mathrm{P}_{\varepsilon}^{s}\left(\left\{f_{n}\right\}_{n=1}^{\infty}, \mu, \delta\right)=\inf \left\{\sum_{i=1}^{\infty} P_{\varepsilon}^{s}\left(\left\{f_{n}\right\}_{n=1}^{\infty}, Z_{i}\right): \mu\left(\bigcup_{i=1}^{\infty} Z_{i}\right) \geq 1-\delta\right\},
$$

where $P_{\varepsilon}^{s}\left(\left\{f_{n}\right\}_{n=1}^{\infty}, Z_{i}\right)$ is defined in Definition 2.2. There exists a critical value of $s$ such that

$$
e_{\mu}^{*}\left(\left\{f_{n}\right\}_{n=1}^{\infty}, \varepsilon, \delta\right)=\left\{s: \mathrm{P}_{\varepsilon}^{s}\left(\left\{f_{n}\right\}_{n=1}^{\infty}, \mu, \delta\right)=0\right\}=\sup \left\{s: \mathrm{P}_{\varepsilon}^{s}\left(\left\{f_{n}\right\}_{n=1}^{\infty}, \mu, \delta\right)=+\infty\right\} .
$$

Define

$$
e_{\mu}^{*}\left(\left\{f_{n}\right\}_{n=1}^{\infty}\right)=\lim _{\delta \rightarrow 0} \lim _{\varepsilon \rightarrow 0} e_{\mu}^{*}\left(\left\{f_{n}\right\}_{n=1}^{\infty}, \varepsilon, \delta\right)
$$

\subsection{NADS}

In this subsection, let $(X, \phi)$ be a NADS, next we introduced NADS's entropies.

Definition 3.7 Let $Z \subset X, s \geq 0, N \in \mathbf{N} \quad$ and $\varepsilon>0$, define

$$
M_{N, \varepsilon}^{s}(\phi, Z)=\inf \sum_{i} \exp \left(-s t_{i}\right)
$$

where the infimum is taken over all finite or countable families $\left\{B_{t_{i}}^{\phi}\left(x_{i}, \varepsilon\right)\right\}$ such that $x_{i} \in X, t_{i} \geq N$ and $\bigcup_{i} B_{t_{i}}^{\phi}\left(x_{i}, \varepsilon\right) \supseteq Z$. The quantity $M_{N, \varepsilon}^{s}(\phi, Z)$ does not decrease as $N$ increase and $\varepsilon$ decreases.

Hence the following limits exist:

$$
\begin{gathered}
M_{\varepsilon}^{s}(\phi, Z)=\lim _{N \rightarrow \infty} M_{N, \varepsilon}^{s}(\phi, Z), \\
M^{s}(\phi, Z)=\lim _{\varepsilon \rightarrow 0} M_{\varepsilon}^{s}(\phi, Z) .
\end{gathered}
$$


Bowen's topological entropy $h_{t o p}^{B}(\phi, Z)$ is defined as a critical value of the parameters $s$, where $M^{s}(\phi, Z)$ jumps from $\infty$ to 0 , i.e.

$$
M^{s}(\phi, Z)= \begin{cases}0 & , s>h_{\text {top }}^{B}(\phi, Z) \\ \infty, & , s<h_{t o p}^{B}(\phi, Z)\end{cases}
$$

Other topological entropy definitions are similar to the discrete case definition.

Definition 3.8 Let $\mu \in M(X), s \geq 0, N \in \mathbf{N}, \varepsilon>0$ and $0<\delta<1$, define

$$
M_{N, \varepsilon}^{s}(\phi, \mu, \delta)=\inf \sum_{i} \exp \left(-s t_{i}\right),
$$

where the infimum is taken over all finite or countable families $\left\{B_{t_{i}}^{\phi}\left(x_{i}, \varepsilon\right)\right\}$ such that $x_{i} \in X, t_{i} \geq N$ and $\mu\left(\bigcup_{i} B_{t_{i}}^{\phi}\left(x_{i}, \varepsilon\right)\right) \geq 1-\delta$. The quantity $M_{N, \varepsilon}^{s}(\phi, \mu, \delta)$ does not decrease as $N$ increase, hence the following limit exist:

$$
M_{\varepsilon}^{s}(\phi, \mu, \delta)=\lim _{N \rightarrow \infty} M_{N, \varepsilon}^{s}(\phi, \mu, \delta) .
$$

Using standard method, we have following is well- defined:

$$
e_{\mu}(\phi, \varepsilon, \delta)=\inf \left\{s: M_{\varepsilon}^{s}(\phi, \mu, \delta)=0\right\}=\sup \left\{s: M_{\varepsilon}^{s}(\phi, \mu, \delta)=+\infty\right\},
$$

defined

$$
e_{\mu}(\phi)=\lim _{\delta \rightarrow 0} \lim _{\varepsilon \rightarrow 0} e_{\mu}(\phi, \varepsilon, \delta)
$$

\section{Proof of Theorem}

\subsection{Proof of Theorem 2.1}

Proposition 4.1 Let $0<\delta<1, \quad \mu \in M(X), \quad\left\{Z_{i}\right\}_{i=1}^{\infty}$ be a family of Borel subsets of $X$ with $\mu\left(\bigcup_{i} Z_{i}\right) \geq 1-\delta$. For any $\varepsilon>0$,

$$
M_{\varepsilon}^{s}\left(\left\{f_{n}\right\}_{n=1}^{\infty}, \mu, \delta\right) \leq \sum_{i=1}^{\infty} M_{\varepsilon}^{s}\left(\left\{f_{n}\right\}_{n=1}^{\infty}, Z_{i}\right) .
$$

Poof For any $\varepsilon>0, N, i \in \mathbb{N}$, there exists $N_{i}>N$ such that

$$
M_{N_{i}, \varepsilon}^{s}\left(\left\{f_{n}\right\}_{n=1}^{\infty}, Z_{i}\right)<M_{\varepsilon}^{s}\left(\left\{f_{n}\right\}_{n=1}^{\infty}, Z_{i}\right)+\frac{\varepsilon}{2^{i}} . \$ \$
$$


Hence, there exists a countable family $\left\{B_{n_{j}^{i}}\left(x_{j}^{i}, \varepsilon\right)\right\}_{j=1}^{\infty}$ such that $n_{j}^{i} \geq N_{i}, \quad x_{j}^{i} \in X$, $\left\{B_{n_{j}^{i}}\left(x_{j}^{i}, \varepsilon\right)\right\}_{j=1}^{\infty} \supseteq Z_{i}$

$$
\sum_{j=1}^{\infty} \exp \left(-s n_{j}^{i}\right)<M_{\varepsilon}^{s}\left(\left\{f_{n}\right\}_{n=1}^{\infty}, Z_{i}\right)+\frac{\varepsilon}{2^{i}}
$$

Since $\mu\left(\bigcup_{i} Z_{i}\right) \geq 1-\delta$, we have $\mu\left(\bigcup_{i \geq 1} \bigcup_{j \geq 1} B_{n_{j}^{i}}\left(x_{j}^{i}, \varepsilon\right)\right) \geq 1-\delta$. Hence

$$
M_{\varepsilon}^{s}\left(\left\{f_{n}\right\}_{n=1}^{\infty}, \mu, \delta\right) \leq \sum_{i \geq 1} \sum_{j \geq 1} \exp \left(-s n_{j}^{i}\right)<\sum_{i=1}^{\infty} M_{\varepsilon}^{s}\left(\left\{f_{n}\right\}_{n=1}^{\infty}, Z_{i}\right) .
$$

Proof (1) Let $Z \subseteq X$ and assume be $0<s<h_{\text {top }}^{B}\left(\left\{f_{n}\right\}_{n=1}^{\infty}, Z\right)$. For any $n \in \mathbf{N}$ and $\varepsilon>0$, let $R=R_{n}\left(\left\{f_{n}\right\}_{n=1}^{\infty}, Z, \varepsilon\right)$ be the largest number so that there is a disjoint family $\left\{\bar{B}_{n}\left(x_{i}, \varepsilon\right)\right\}_{i=1}^{R}$ with $x_{i} \in Z$. Then it is easy to see that for any $\delta>0$,

$$
\bigcup_{i=1}^{R} \bar{B}_{n}\left(x_{i}, 2 \varepsilon+\delta\right) \supseteq Z,
$$

which implies that

$$
M_{n, 2 \varepsilon+\delta}^{s}\left(\left\{f_{n}\right\}_{n=1}^{\infty}, Z\right) \leq R \cdot \exp (-n s) \leq P_{n, \varepsilon}^{s}\left(\left\{f_{n}\right\}_{n=1}^{\infty}, Z\right)
$$

for any $s \geq 0$, and hence $M_{2 \varepsilon+\delta}^{s}\left(\left\{f_{n}\right\}_{n=1}^{\infty}, Z\right) \leq P_{\varepsilon}^{s}\left(\left\{f_{n}\right\}_{n=1}^{\infty}, Z\right)$, we have $M_{2 \varepsilon+\delta}^{s}\left(\left\{f_{n}\right\}_{n=1}^{\infty}, Z\right) \leq \mathrm{P}_{\varepsilon}^{s}\left(\left\{f_{n}\right\}_{n=1}^{\infty}, Z\right)$. Since $0<s<h_{\text {top }}^{B}\left(\left\{f_{n}\right\}_{n=1}^{\infty}, Z\right)$, we have $M^{s}\left(\left\{f_{n}\right\}_{n=1}^{\infty}, Z\right)=\infty$ and thus $M_{2 \varepsilon+\delta}^{s}\left(\left\{f_{n}\right\}_{n=1}^{\infty}, Z\right) \geq 1$ when $\varepsilon$ and $\delta$ are small enough. Hence $\mathrm{P}_{\varepsilon}^{s}\left(\left\{f_{n}\right\}_{n=1}^{\infty}, Z\right) \geq 1$ and $h_{\text {top }}^{P}\left(\left\{f_{n}\right\}_{n=1}^{\infty}, Z, \varepsilon\right) \geq s$ when $\varepsilon$ is small.

Therefore $h_{\text {top }}^{P}\left(\left\{f_{n}\right\}_{n=1}^{\infty}, Z\right)=\lim _{\varepsilon \rightarrow 0} h_{\text {top }}^{P}\left(\left\{f_{n}\right\}_{n=1}^{\infty}, Z, \varepsilon\right) \geq s$.

This implies that $h_{\text {top }}^{B}\left(\left\{f_{n}\right\}_{n=1}^{\infty}, Z\right) \leq h_{\text {top }}^{P}\left(\left\{f_{n}\right\}_{n=1}^{\infty}, Z\right)$.

(2) Denote

$$
\bar{h}_{\mu}^{K}\left(\left\{f_{n}\right\}_{n=1}^{\infty}, \varepsilon, \delta\right)=\limsup _{n \rightarrow \infty} \frac{1}{n} \log r_{n}(\mu, \varepsilon, \delta)
$$

then $\bar{h}_{\mu}^{K}\left(\left\{f_{n}\right\}_{n=1}^{\infty}\right)=\lim _{\delta \rightarrow 0} \lim _{\varepsilon \rightarrow 0} \bar{h}_{\mu}^{K}\left(\left\{f_{n}\right\}_{n=1}^{\infty}, \varepsilon, \delta\right)$. 
We first prove that

$$
\bar{e}_{\mu}\left(\left\{f_{n}\right\}_{n=1}^{\infty}, \varepsilon, \delta\right) \leq \bar{h}_{\mu}^{K}\left(\left\{f_{n}\right\}_{n=1}^{\infty}, \varepsilon, \delta\right)
$$

for any $0<\delta<1$ and $\varepsilon>0$, using like-Huasdorff dimension method. For any $s>\bar{h}_{\mu}^{K}\left(\left\{f_{n}\right\}_{n=1}^{\infty}, \varepsilon, \delta\right)$ and $Z \in X_{\mu, \delta}$, let $F$ is a $(n, \varepsilon, Z)$-spanning set, then

$$
\left.R_{n, \delta}^{s}\left(\left\{f_{n}\right\}_{n=1}^{\infty}, \mu, \delta\right) \leq \sum_{x \in F} \exp (-s n)=\# F \cdot \exp (-s n)\right\}
$$

which follows that

$$
R_{n, \varepsilon}^{s}\left(\left\{f_{n}\right\}_{n=1}^{\infty}, \mu, \delta\right) \leq \exp (-s n) \cdot \inf _{Z \in X_{\mu, \delta}} r_{n}(\varepsilon, Z) .
$$

Hence

$$
R_{n, \varepsilon}^{s}\left(\left\{f_{n}\right\}_{n=1}^{\infty}, \mu, \delta\right) \leq \exp (-s n) \cdot r_{n}(\mu, \varepsilon, \delta)=e^{-n\left(s-\frac{1}{n} \log r_{n}(\mu, \varepsilon, \delta)\right)} .
$$

Since $\bar{h}_{\mu}^{K}\left(\left\{f_{n}\right\}_{n=1}^{\infty}, \varepsilon, \delta\right)=\limsup _{n \rightarrow \infty} \frac{1}{n} \log r_{n}(\mu, \varepsilon, \delta)<s$, we have

$$
\limsup _{n \rightarrow \infty} R_{n, \varepsilon}^{s}\left(\left\{f_{n}\right\}_{n=1}^{\infty}, \mu, \delta\right)=0 .
$$

For $s>\bar{h}_{\mu}^{K}\left(\left\{f_{n}\right\}_{n=1}^{\infty}, \varepsilon, \delta\right)$ we get $\bar{R}_{\varepsilon}^{s}\left(\left\{f_{n}\right\}_{n=1}^{\infty}, \mu, \delta\right)=0$ and $\bar{e}_{\mu}\left(\left\{f_{n}\right\}_{n=1}^{\infty}, \varepsilon, \delta\right) \leq s$.

Hence $\bar{e}_{\mu}\left(\left\{f_{n}\right\}_{n=1}^{\infty}, \varepsilon, \delta\right) \leq \bar{h}_{\mu}^{K}\left(\left\{f_{n}\right\}_{n=1}^{\infty}, \varepsilon, \delta\right)$.

Next we prove $\bar{e}_{\mu}\left(\left\{f_{n}\right\}_{n=1}^{\infty}, \varepsilon, \delta\right) \geq \bar{h}_{\mu}^{K}\left(\left\{f_{n}\right\}_{n=1}^{\infty}, \varepsilon, \delta\right)$ for any $0<\delta<1$ and $\varepsilon>0$ by showing $\bar{h}_{\mu}^{K}\left(\left\{f_{n}\right\}_{n=1}^{\infty}, \varepsilon, \delta\right) \leq s$ whenever $s>\bar{e}_{\mu}\left(\left\{f_{n}\right\}_{n=1}^{\infty}, \varepsilon, \delta\right)$. For such a $s$, we have $\bar{R}_{\varepsilon}^{s}\left(\left\{f_{n}\right\}_{n=1}^{\infty}, \mu, \delta\right)=0$. Then there exists $N \in \mathbf{N}$ such that $R_{n, \varepsilon}^{s}\left(\left\{f_{n}\right\}_{n=1}^{\infty}, \mu, \delta\right)<1$ for any $n \geq N$. Fix $n \geq N$, we can find a finite family $\left\{B_{n}\left(x_{i}, \varepsilon\right)\right\}_{i \in I}$ such that $x_{i} \in X$,

$$
\mu\left(\bigcup_{i \in I} B_{n}\left(x_{i}, \varepsilon\right)\right) \geq 1-\delta \text { and } \# I \cdot e^{-s n}<1
$$

So $r_{n}(\mu, \varepsilon, \delta) \leq e^{s n}$ for any $n \geq N$. Hence $\bar{h}_{\mu}^{K}\left(\left\{f_{n}\right\}_{n=1}^{\infty}, \varepsilon, \delta\right) \leq s$. 
(3) The proof of (3) is similar to (2).

(4) The proof of (4) is a consequence of definition.

(5) We first show that $e_{\mu}\left(\left\{f_{n}\right\}_{n=1}^{\infty}\right) \leq e_{\mu}^{*}\left(\left\{f_{n}\right\}_{n=1}^{\infty}\right)$. Let $s<e_{\mu}\left(\left\{f_{n}\right\}_{n=1}^{\infty}\right), \quad 0<\delta<1$ and $\left\{Z_{i}\right\}_{i=1}^{\infty}$ be a family of Borel subsets of $X$ with $\mu\left(\bigcup_{i=1}^{\infty} Z_{i}\right) \geq 1-\delta$. For any $i, n \in \mathbf{N}$ and $\varepsilon>0$, let $R_{n}^{i}=R\left(Z_{i}, \varepsilon\right)$ be the largest number such that there is a disjoint family $\left\{\bar{B}_{n}\left(x_{j}^{i}, \varepsilon\right)\right\}_{j=1}^{R_{n}^{i}}$ with $x_{j}^{i} \in Z_{i}$. Then we can verify that for any $\theta>0$

$$
\left\{B_{n_{j}^{i}}\left(x_{j}^{i}, 2 \varepsilon+\theta\right)\right\} \supseteq Z_{i} .
$$

It following that $M_{n, 2 \varepsilon+\theta}^{s}\left(\left\{f_{n}\right\}_{n=1}^{\infty}, Z_{i}\right) \leq R_{n}^{i} \cdot e^{-s n} \leq P_{n, \varepsilon}^{s}\left(\left\{f_{n}\right\}_{n=1}^{\infty}, Z_{i}\right)$ and $M_{2 \varepsilon+\theta}^{s}\left(\left\{f_{n}\right\}_{n=1}^{\infty}, Z_{i}\right) \leq \mathrm{P}_{\varepsilon}^{s}\left(\left\{f_{n}\right\}_{n=1}^{\infty}, Z_{i}\right)$. Therefore, by the Proposition4.1, we have $M_{2 \varepsilon+\theta}^{s}\left(\left\{f_{n}\right\}_{n=1}^{\infty}, \mu, \delta\right) \leq \mathrm{P}_{\varepsilon}^{s}\left(\left\{f_{n}\right\}_{n=1}^{\infty}, \mu, \delta\right)$. As $s<e_{\mu}\left(\left\{f_{n}\right\}_{n=1}^{\infty}\right)$, we can get $s<e_{\mu}\left(\left\{f_{n}\right\}_{n=1}^{\infty}, 2 \varepsilon+\theta, \delta\right)$ when $\varepsilon, \theta, \delta$ are small enough. This implies that $M_{2 \varepsilon+\theta}^{s}\left(\left\{f_{n}\right\}_{n=1}^{\infty}, \mu, \delta\right)=\infty$ and thus $\mathrm{P}_{\varepsilon}^{s}\left(\left\{f_{n}\right\}_{n=1}^{\infty}, \mu, \delta\right)=\infty$. Therefore, it can be deduced that $e_{\mu}^{*}\left(\left\{f_{n}\right\}_{n=1}^{\infty}\right) \geq s$. So the desired inequality holds.

Now we proved that $e_{\mu}^{*}\left(\left\{f_{n}\right\}_{n=1}^{\infty}\right)=\lim _{\varepsilon \rightarrow 0} \lim _{\delta \rightarrow 0} \inf _{Z \in X_{\mu, \delta}} h_{t o p}^{P}\left(\left\{f_{n}\right\}_{n=1}^{\infty}, Z, \varepsilon\right)$.

Let $e_{\mu}^{*}\left(\left\{f_{n}\right\}_{n=1}^{\infty}\right)>s$, then there exists $\varepsilon^{\prime}, \delta^{\prime}>0$ such that $e_{\mu}^{*}\left(\left\{f_{n}\right\}_{n=1}^{\infty}, \varepsilon, \delta\right) \geq s$ for any $\varepsilon \in\left(0, \varepsilon^{\prime}\right)$ and $\delta \in\left(0, \delta^{\prime}\right)$. Thus $\mathrm{P}_{\varepsilon}^{s}\left(\left\{f_{n}\right\}_{n=1}^{\infty}, \mu, \delta\right)=\infty$.

For any $Z \in X_{\mu, \delta}$ and any $\left\{Z_{i}\right\}_{i \geq 1}$ with $\bigcup_{i=1}^{\infty} Z_{i} \supseteq Z$, we have $\mu\left(\bigcup_{i=1}^{\infty} Z_{i}\right) \geq 1-\delta$. It follows from $\mathrm{P}_{\varepsilon}^{s}\left(\left\{f_{n}\right\}_{n=1}^{\infty}, \mu, \delta\right)=\infty$ that $\sum_{i=1}^{\infty} P_{\varepsilon}^{s}\left(\left\{f_{n}\right\}_{n=1}^{\infty}, Z_{i}\right)=\infty$.

$\operatorname{SoP}_{\varepsilon}^{s}\left(\left\{f_{n}\right\}_{n=1}^{\infty}, Z\right)=\infty$, which gives that $h_{\text {top }}^{P}\left(\left\{f_{n}\right\}_{n=1}^{\infty}, Z, \varepsilon\right) \geq s$.

On the other hand, let $s<\lim _{\varepsilon \rightarrow 0} \lim _{\delta \rightarrow 0} \inf _{Z \in X} h_{\mu, \delta}^{P}\left(\left\{f_{n}\right\}_{n=1}^{\infty}, Z, \varepsilon\right)$. Then there exist $\varepsilon^{\prime}$, $\delta^{\prime}>0$ such that $h_{t o p}^{P}\left(\left\{f_{n}\right\}_{n=1}^{\infty}, Z, \varepsilon\right)>s$ for any $\varepsilon \in\left(0, \varepsilon^{\prime}\right), \delta \in\left(0, \delta^{\prime}\right)$ and 
$Z \in X_{\mu, \delta}$. Thus, we have $\mathrm{P}_{\varepsilon}^{s}\left(\left\{f_{n}\right\}_{n=1}^{\infty}, Z\right)=\infty$. Fix $\quad\left\{Z_{i}\right\}_{i \geq 1}$ with $\mu\left(\bigcup_{i=1}^{\infty} Z_{i}\right) \geq 1-\delta$ and write $Z=\bigcup_{i=1}^{\infty} Z_{i}$, then $Z \in X_{\mu, \delta}$. So $\sum_{i=1}^{\infty} P_{\varepsilon}^{s}\left(\left\{f_{n}\right\}_{n=1}^{\infty}, Z_{i}\right)=\infty$, which yields that $\mathrm{P}_{\varepsilon}^{s}\left(\left\{f_{n}\right\}_{n=1}^{\infty}, \mu, \delta\right)=\infty$. Furthermore, we can get $e_{\mu}^{*}\left(\left\{f_{n}\right\}_{n=1}^{\infty}, \varepsilon, \delta\right) \geq s$ and $e_{\mu}^{*}\left(\left\{f_{n}\right\}_{n=1}^{\infty}\right) \geq s$.

\subsection{Proof of Theorem 2.2}

Proposition 4.2 For $\mu \in M(X)$, it holds that

$$
\underline{h}_{\mu}\left(\left\{f_{n}\right\}_{n=1}^{\infty}\right) \leq e_{\mu}\left(\left\{f_{n}\right\}_{n=1}^{\infty}\right) \leq \inf \left\{h_{t o p}^{B}\left(\left\{f_{n}\right\}_{n=1}^{\infty}, K\right): \mu(K)=1\right\} .
$$

Proof The second inequality is a direct consequence of the definition and we only deduce the first one. For $s>0$ with $\underline{h}_{-\mu}\left(\left\{f_{n}\right\}_{n=1}^{\infty}\right)>s$. By a standard procedure, there exist $A \subset X$ with $\mu(A)>0$ and $N \in \mathbf{N}$ such that

$$
\mu\left(B_{n}(x, \varepsilon)\right)<e^{-s n}, \forall x \in A, n \geq N
$$

Pick $\delta \in(0, \mu(A))$. Let $\left\{B_{n_{i}}\left(x_{i}, \frac{\varepsilon}{2}\right)\right\}_{i \in I}$ be a countable family such that $n_{i} \geq N$, $x_{i} \in X$ and $\mu\left(\bigcup_{i \in I} B_{n_{i}}\left(x_{i}, \frac{\varepsilon}{2}\right)\right) \geq 1-\delta$ that intersects $A$, if taking $y_{i} \in B_{n_{i}}\left(x_{i}, \frac{\varepsilon}{2}\right) \cap A$, then one has $B_{n_{i}}\left(x_{i}, \frac{\varepsilon}{2}\right) \subseteq B_{n_{i}}\left(y_{i}, \varepsilon\right)$ and thus

$$
\mu\left(B_{n_{i}}\left(x_{i}, \frac{\varepsilon}{2}\right)\right) \leq \mu\left(B_{n_{i}}\left(y_{i}, \varepsilon\right)\right) \leq e^{-s n_{i}}
$$

Then we have

$$
\begin{gathered}
\sum_{i \in I} e^{-s n_{i}} \geq \sum_{i \in I} \mu\left(B_{n_{i}}\left(y_{i}, \varepsilon\right) \cap A\right) \geq \sum_{i \in I} \mu\left(B_{n_{i}}\left(x_{i}, \frac{\varepsilon}{2}\right) \cap A\right) \\
\geq \mu\left(\bigcup_{i \in I} B_{n_{i}}\left(x_{i}, \frac{\varepsilon}{2}\right) \cap A\right)=\mu(A)>0
\end{gathered}
$$

Hence $M_{\frac{\varepsilon}{2}}^{s}\left(\left\{f_{n}\right\}_{n=1}^{\infty}, \mu, \delta\right) \geq M_{N, \frac{\varepsilon}{2}}^{s}\left(\left\{f_{n}\right\}_{n=1}^{\infty}, \mu, \delta\right) \geq \mu(A)$. By Bowen's definition, we can derive that $e_{\mu}\left(\left\{f_{n}\right\}_{n=1}^{\infty}, \frac{\varepsilon}{2}, \delta\right) \geq s$ and moreover $\underline{h}_{-\mu}\left(\left\{f_{n}\right\}_{n=1}^{\infty}\right) \leq e_{\mu}\left(\left\{f_{n}\right\}_{n=1}^{\infty}\right)$. 
Definition 4.3 Let $\mu \in M(X)$. The NADS $(X, \phi)$ measure-theoretical lower entropies of $\mu$ is defined by

$$
\underline{h}_{\mu}(\phi)=\int_{\underline{h}_{\mu}}(\phi, x) d \mu(x)
$$

where

$$
\underline{h}_{\mu}(\phi, x)=\lim _{\varepsilon \rightarrow 0} \liminf _{t \rightarrow \infty}-\frac{1}{t} \log \mu\left(B_{t}^{\phi}(x, \varepsilon)\right) .
$$

Lemma 4.4 ([5,theorem1.4]) Let $\left(X,\left\{f_{n}\right\}_{n=1}^{\infty}\right)$ be a NADDS. If $K \subseteq X$ is non-empty and compact, then

$$
h_{\text {top }}^{B}\left(\left\{f_{n}\right\}_{n=1}^{\infty}, K\right)=\sup \left\{\frac{h}{\mu}_{\mu}\left(\left\{f_{n}\right\}_{n=1}^{\infty}\right): \mu \in M(X), \mu(K)=1\right\} .
$$

Proof By the Proposition, we have

$$
\begin{aligned}
\sup \left\{\underline{h}_{\mu}\left(\left\{f_{n}\right\}_{n=1}^{\infty}\right):\right. & \mu \in M(X), \mu(K)=1\} \leq \sup \left\{e_{\mu}\left(\left\{f_{n}\right\}_{n=1}^{\infty}\right): \mu \in M(X), \mu(K)=1\right\} \\
& \leq \inf \left\{h_{\text {top }}^{B}\left(\left\{f_{n}\right\}_{n=1}^{\infty}, K\right): \mu \in M(X), \mu(K)=1\right\}
\end{aligned}
$$

Combining with lemma,

$$
h_{\text {top }}^{B}\left(\left\{f_{n}\right\}_{n=1}^{\infty}, K\right)=\sup \left\{\underline{h}_{\mu}\left(\left\{f_{n}\right\}_{n=1}^{\infty}\right): \mu \in M(X), \mu(K)=1\right\}
$$

the conclusion can be proved.

Using the same proof method of Theorem 2.1 and, Theorem 2.2, we have result of Theorem 2.3 and Theorem 2.4. 


\section{References}

[1] Adler, R., Konheim, A. and McAndrew, M. (1965). Topological entropy. Trans. Amer. Math. Soc, 114, pp.309-319.

[2] Bowen, R. (1973). Topological entropy for noncompact sets. Trans. Amer. Math. Soc, 184, pp.125-136.

[3] Walters, P. (1981). An Introduction to Ergodic Theory. Springer, Berlin.

[4] Kolyada, S. and Snoha, L. (1996). Topological entropy of nonautonomous dynamical systems. Random. Dyn. 4, pp. 205-233

[5] Xu, L. and Zhou, X. (2018). Variational principles for entropies of nonautonomous dynamical systems. J. Dyn. Differ. Equ. 30, pp. 1053-1062.

[6] Feng, D. and Huang, W. (2012). Variational principle for topological entropies of subsets. J. Funct. Anal. 263, pp.2228-2254.

[7] Zhou, X. (2016). A formula of conditional entropy and some applications. Discrete Contin. Dyn. Syst. 36, pp.4063-4075.

[8] Brin, M. and Katok, A. (1999). On local entropy. I. Math. Phys. Anal. Geom. 2, pp. 323-415.

[9] Wang, T. (2021). Some note on tological and measure-theoretic entropy. Qual. Theory Dyn.Syst. 20.

[10] Kawan, C. (2013). Metric entropy of nonautonomous dynamical systems. Nonauton. Dyn. Syst. 1, pp.26-52.

[11] Kolyada, S., Misiurewicz, M. and Snoha, L. (1990). Topological entropy of nonautonomous piecewise monotone dynamical systems on the interval. Fund. Math. 160, pp.161-181.

[12] Zhu, Y., Liu, Z. and Zhang, W. (2012). Entropy of nonautonomous dynamical systems. J. Korean Math. 49, pp.165-185. 